\title{
Collisional versus laser driven ionization in metal clusters
}

\author{
P.-G. Reinhard ${ }^{1}$ and E. Suraud ${ }^{2} * *$ \\ ${ }^{1}$ Institut für theoretische Physik, Universität Erlangen, Staudtstrasse 7, D-91058 Erlangen, Germany \\ ${ }^{2}$ Laboratoire de Physique Quantique,Université P. Sabatier,118 Route de Narbonne, 31062 Toulouse, \\ cedex, France
}

**Author to whom correspondence should be addressed.

Received: 10 May 2000 / Accepted: 25 July 2000 / Published: 14 November 2000

\begin{abstract}
We compare the capabilities of rapid highly charged projectiles and intense femtosecond lasers to ionize simple metal clusters while leaving as little intrinsic excitation as possible in the residue. We show that both excitation mechanisms are able to produce highly charged clusters. The deposited excitation energies increase with ionization but with different trends. Cold ionization, corresponding to moderate deposited excitation energy, is better attained with ionic projectiles for low charge states, and with lasers for high charge states.
\end{abstract}

Keywords: Metal clusters, intense laser, ionic collisions, density functional theory.

While the optical response has been a key tool of investigation of the structure and linear dynamics of metal clusters [1-4], the technological developments of the last few years have oriented investigations towards the study of highly excited electronic states. There are in particular two classes of excitations which have raised much interest: excitation by highly charged ionic projectiles [5] and irradiation by intense lasers [6]. Both excitation processes deliver a short and violent electro-magnetic pulse to the cluster, whose immediate response is predominantly of electronic nature due to the short time scales involved (at most a few tens of fs). While the excitation mechanisms differ in the frequency selectivity they provide (laser deliver a highly selective frequency signal, while collisions deliver just a sharp pulse covering many frequencies), the electron response very soon converts the initial excitation into collective plasmon oscillation, electron emission and intrinsic "heat" The further evolution of the system, on the picosecond time scale, consists in a Coulomb explosion as soon as the ionization of the cluster exceeds a critical value. This critical value depends sensitively on the temperature of the cluster and that is why one aims at a cold ionization [7]. 
These fast excitation processes differ from collisions with slow ions, in which the electronic response is fully adiabatic, or from irradiation by nanosecond lasers, in which ions have time to be heated up significantly in the course of the excitation itself. On the contrary, the "new" fast and violent perturbations (rapid multi-charged ion or intense fs lasers) lead into the much different regime of nonadiabatic electronic response without direct ionic excitation. In particular, energetic ionic collisions have been propounded as a way to produce highly ionized, but cold, clusters [7], namely clusters in which only little excitation energy is deposited, which allows to circumvent the problems of low fluency ns lasers. But it turns out that fs lasers also allow to deposit much less heat than ns lasers and thus offer an interesting alternative. Such "cold" ionization is of crucial importance for a deeper understanding of fission or fragmentation [7,8] because the stability of the clusters is extended to higher charge states when heating can be avoided. It is thus desirable to explore the relative merits of both techniques with respect to ionization and "excitation" energy deposit. It is the aim of this paper to investigate this question theoretically and in a quantitative way, taking as examples the systems typically used in experiments [5].

We describe the electronic dynamics in terms of density functional theory [9] at the level of the time dependent local density approximation (TDLDA) following here several former investigations on similar physical situations [10-17]. It was furthermore shown in [18] that the TDLDA provides a sufficient degree of sophistication for theoretical studies of the impact of fs laser pulses. The same holds for the (in that respect not so different) short Coulomb pulses from the colliding ion, as was also discussed at length in [13] and in calculations involving similar theoretical methods [19]. Technical details, both at formal and computational levels, about ingredients and performances of our approach can be found in [10]. In the present investigation we use the functional of [20] to treat electronic exchange and correlations. What the ionic background is concerned, we can neglect effects from ionic motion in view of the short time scales considered here (excitation times $8.50 \mathrm{fs}$ ). As a further simplification we neglect the detailed ionic structure of the cluster and treat the ions at the level of the jellium approximation, as in our previous investigation of both ionic collisions [13] and laser irradiations [11]. This has the particular advantage to allow direct comparisons to other calculations performed for similar situations [17,19]. We actually use here the jellium background with soft surface which has been shown to provide a quantitatively correct electronic response in the linear domain, see e.g. [22].

The actual solution of the TDLDA equations employs axial symmetry which is exact in the case of laser excitations and a good approximation in the case of ion collisions (as we will see). The laser is modelled as an oscillating dipole field with a cosine ${ }^{2}$ profile. The ion collision is modelled by the (axially averaged) Coulomb field of the ion passing by on a straight line with given velocity vI and impact parameter $b$ [13]. At the high projectile velocities considered here this turns out to be a very good approximation [13,19,21]. Wave-functions and fields are represented on a two-dimensional axial grid in coordinate space. A proper choice of grid parameters (size and mesh) allows to quantify any electronic excitation relevant for our problem such as for example electron emission [11,13]. The method also ensures a pertinent description of electron transfer into the vicinity of the projectile $[13,17,19]$. Note that we have taken care to use an appropriate local pseudopotential to arrange the correct representation of the projectile ion on the grid, following [17,19]. The stationary ground state of the cluster is obtained by an accelerated gradient step. The time evolution is done with the Crank- 
Nicholson scheme. For more details of the numerical implementation and further references see [10].

The key observables in the context of the present discussion are ionization and its relation to the deposited excitation energy in the cluster. We shall thus focus the present analysis on these two quantities. Ionization can be easily acceeded in TDLDA by computing the number of ${ }^{0}$ electrons which have finally escaped the numerical box, i.e.

$$
\mathrm{N}_{\mathrm{esc}}(\mathrm{t})=\mathrm{N}(\mathrm{t}=0)-\mathrm{d}^{3} \mathrm{r} \rho(\mathbf{r}, \mathrm{t})
$$

where $\rho(\mathbf{r}, t)$ is the electron density inside the box. We will not discuss the detailed time evolution, as was done in $[10,13]$. Here we concentrate on the asymptotic value $\mathrm{N}_{\text {esc }} \bowtie \mathrm{N}_{\text {esc }}(\mathrm{t} \bullet \boldsymbol{\bullet})$ which is evaluated actually after a finite but sufficiently long time (50 fs for fast collisions, $250 \mathrm{fs}$ for slow collisions, and 200 fs for laser excitation). The internal excitation energy deposited in the electron cloud is defined as

$$
\mathrm{E}_{\text {int }}=\mathrm{E}_{\mathrm{kin}}-\mathrm{E}^{0} \mathrm{ETF}, \mathrm{kin}-\mathrm{E}_{\mathrm{coll}, \mathrm{kin}}
$$

where Ekin is the total kinetic energy according to the Kohn-Sham scheme. The second term E0ETF,kin subtracts the instantaneous and local kinetic energy of the zero-temperature ground state at given density $\rho(r, t)$. It is computed in Extended Thomas-Fermi (ETF) approximation which provides a very efficient and reliable estimate [23]. The last term stands for the collective kinetic energy, $\mathrm{d} 3 \mathrm{r}$ $j 2 / 2 \rho(r, t)(j(r)$ being the local current), contained in the coherent dipole oscillations of the cluster. The intrinsic energy Eint is thus gathering the non collective part of the kinetic energy, once subtracted the zero temperature contribution from Pauli pressure (ETF part). Again, we consider here only the asymptotic value Eint $₫ \operatorname{Eint}(\mathrm{t} \bullet$ ), as in the case of ionization.

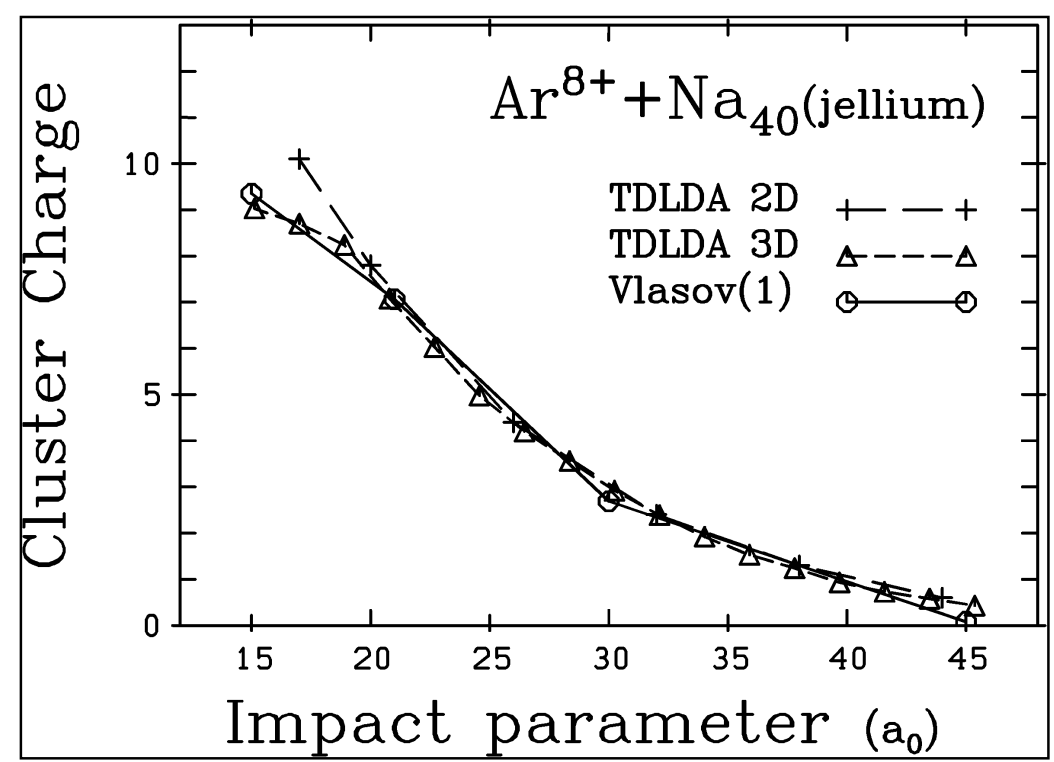

Figure 1. Ionization as a function of impact parameter for collisions of $\mathrm{Ar}^{8+}$ on $\mathrm{Na}_{40}$ at $\mathrm{v}_{\mathrm{I}}=\mathrm{v}_{\mathrm{F}}$ in various calculations: 3D TDLDA of [19], 3D Vlasov of [17] and our 2D TDLDA. Note that the jellium qbackground has been here taken in its sharp version (contrarily to all our other calculations) for a proper comparison with the results of $[17,19]$. 
As a first test, we have compared our 2D method with fully three-dimensional TDLDA calculations [19] and with the semi-classical Vlasov-TDLDA approach [17]. Test case was Ar8+ at $80 \mathrm{keV}$ kinetic energy colliding with a Na40 cluster. The choice of such parameters is motivated by experiments [5] as well as by recent computations on that system [17,19]. Figure 1 compares the results of the three methods for $\mathrm{Na}_{40}$ as a function of impact parameter $\mathrm{b}$ defined as the distance between the cluster and the projectile trajectory (which is basically a straight line at the high projectile velocities considered here). The agreement between the different calculations is striking. It proves the validity of our $2 \mathrm{D}$ treatment for ion collisions as well as the validity of a Vlasov approach for that case. Slight differences can be spotted for small impact parameters. But this is harmless because only peripheral collisions are properly described in such a formalism with frozen cluster ions $[13,17,19]$.

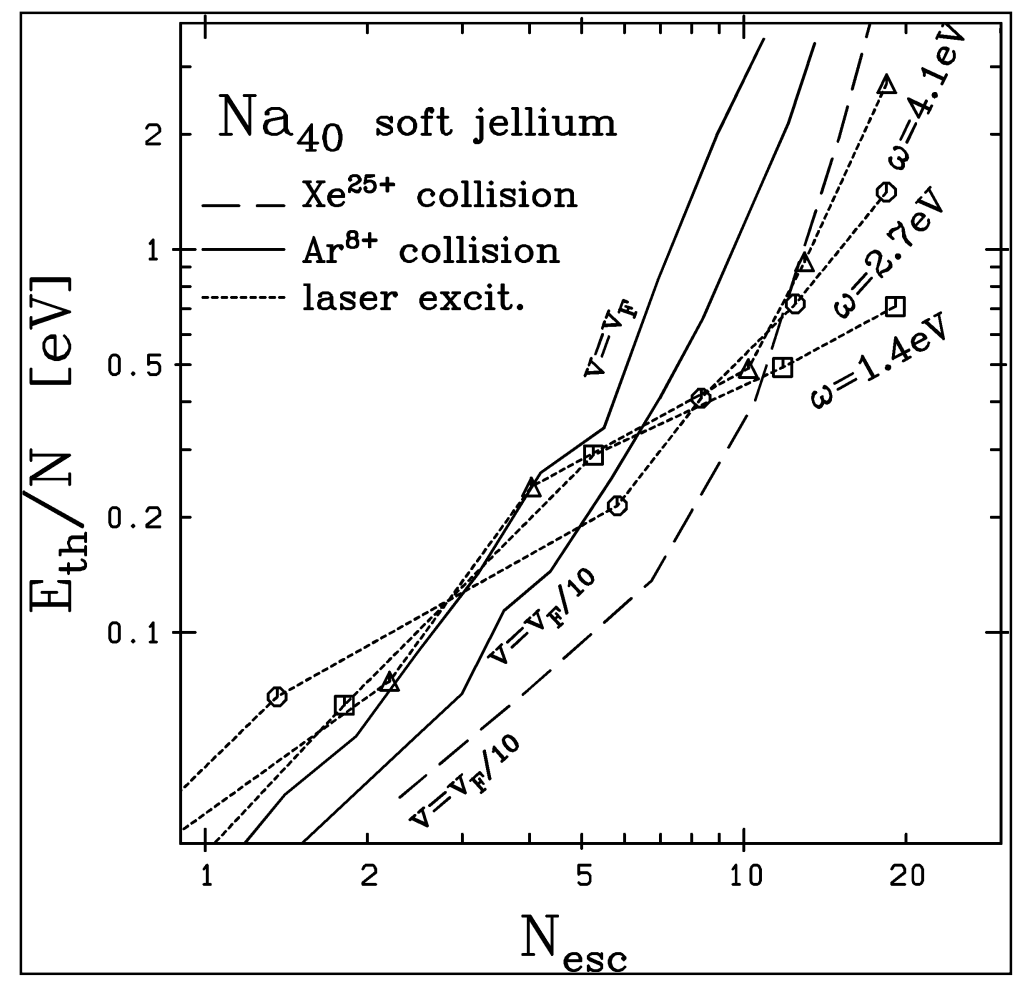

Figure 2. Intrinsic energy $\mathrm{E}_{\mathrm{int}} / \mathrm{N}$ per bound electron as a function of ionization $\mathrm{N}_{\mathrm{esc}}$ for various collisions and various laser pulses, as indicated on the figure. See text for details.

In a second step, we have employed our TDLDA method to scan systematically ion collisions and laser excitations under widely varying conditions. Test case remains $\mathrm{Na} 40$. Ion collisions have been considered with $\mathrm{Ar}^{8+}$ at $\mathrm{v}_{\mathrm{I}}=\mathrm{v}_{\mathrm{F}}$ and $\mathrm{v}_{\mathrm{F}} / 10$ ( $\mathrm{v}_{\mathrm{F}}$ is the Fermi velocity of cluster's electrons) as well as with $\mathrm{Xe}^{25+}$ at $\mathrm{v}_{\mathrm{F}} / 10$. In these collisional cases, the impact parameter $b$ of the projectile, has been varied from 15 to 70 a 0 . This allows to scan a wide range of ionization states (between virtually 0 and about half the total cluster charge) within remaining safely in the regime of peripheral reactions for which our schematic treatment of ionic background is justified [13,17,19]. What the lasers are concerned, we aim at short pulses, first, because we expect most efficient excitations there, and second, because we use frozen ions and the laser pulse length should thus not interfere with ionic time scales. We hence use a laser pulse of 50 fs FWHM. Previous investigations $[11,12,24]$ have shown that the Mie plasmon plays a key role in the ionization pattern. We have thus chosen three representative 
laser frequencies, below $(\omega=1.4 \mathrm{eV})$, at $(\omega=2.7 \mathrm{eV})$ and above $(\omega=4.1 \mathrm{eV})$ the Mie plasmon resonance (around $2.6 \mathrm{eV}$ for the considered cluster). Finally, the laser intensities are varied in the range $\mathrm{I}-10^{9}$ to $10^{14} \mathrm{~W} / \mathrm{cm}^{2}$, which again corresponds to span values of $\mathrm{N}_{\mathrm{esc}}$ up to half ionization of the cluster. It is noteworthy that the maximum intensity of $10^{14} \mathrm{~W} / \mathrm{cm}^{2}$ used here stays safely below the critical intensity where core electrons and other violent dynamical effects come into play in sodium [12]. The results of our systematic scan are displayed in Figure 2 where we have plotted the intrinsic energy $E_{i n t} / \mathrm{N}$ per bound electron as a function of ionization $\mathrm{N}_{\mathrm{esc}}$ for the various excitation mechanisms described above. The number of bound electrons is defined here as the complement of $\mathrm{N}_{\text {esc }}$, i.e. $\mathrm{N}=40-\mathrm{N}_{\text {esc }}$. We see that the trend is the same in all cases: $\mathrm{E}_{\text {int }} / \mathrm{N}$ strongly increases with $\mathrm{N}_{\text {esc }}$. This shows that the heating up of the remaining cluster is hardly avoidable as soon as one aims at producing high ionization states. However, the functional form of the growth is different for the both mechanisms. All laser excitations follow approximately $\mathrm{E}_{\mathrm{int}} / \mathrm{N} \alpha \mathrm{N}_{\mathrm{esc}}{ }^{1}$ whereas all ion collisions seem to gather around a growth $\alpha \mathrm{N}_{\mathrm{esc}}{ }^{2}$. Mind that Figure 2 is displayed in a double logarithmic scale, so that the observed differences are, in fact, rather large.

There are also large differences within the various ionic collisions. We find that the ionization grows with projectile charge, as already observed in [17] for collisions at $\mathrm{v}_{\mathrm{I}}=\mathrm{v}_{\mathrm{F}}$. We recover here a similar trend for $v_{I}=v_{F} / 10$. We also see that the lower velocity $v_{I}=v_{F} / 10$ is more favourable to cold ionization. Thus, the optimum choice is a very highly charged ion at moderate velocity. Lasers, on the other hand, behave in a less systematic way. Except for very high ionization states, which correspond to very large intensities here, there are no systematic shifts between the various frequencies nor significantly different trends. But this does not mean that all the three laser cases behave the same in every respect. For example, for the same intensity $I$ there can be several orders of magnitude more yield when $\omega$ is on resonance than when it is off resonance. But here we compare the net energy balance $E_{i n t} / N$ versus $N_{\text {esc }}$ where the intensity is tuned in each case to provide the same yield $\mathrm{N}_{\text {esc }}$. And this net energy balance is found to be insensitive to the details of the laser excitation.

The most interesting outcome from Figure 2 concerns the difference between laser and ionic excitations. The general trend is clear. Ionic collisions are more efficient for "cold" ionization with moderate deposited excitation energy (lower left corner of Figure 2) at the side of low ionization, while laser excitation becomes competitive at higher $\mathrm{N}_{\mathrm{esc}}$ and even takes the lead for very high $\mathrm{N}_{\mathrm{esc}}$ at around stripping one quarter of the valence electrons of the cluster (upper right corner of Figure 2). The break even point between ion collisions and lasers can be shifted further upwards by proceeding to a higher charge state of the projectile ion. But that reaches soon the limits of affordable ion sources and requires a large heavy-ion accelerator. We hence arrive at the interesting conclusion that today's lasers deliver excitation conditions which are surprisingly similar to ion collisions, at least in terms of ionization and associated excitation energy deposits. It even seems that lasers offer better possibilities to attain very high ionization states without depositing too much heat in the system.

To conclude, we have made a systematic exploration of the ionization and excitation energy deposited in a sodium cluster either bombarded by rapid highly charged ions or irradiated by short and intense laser beams. We have seen that today's lasers offer excitation conditions comparable to the ones attained in ionic collisions, however, with slightly different bias: ion collisions remain unbeatable for low and moderate ionization (up to about $25 \%$ ) while lasers take the lead for higher ionization. As a side-result we have found that the two dimensional (axial) TDLDA and the semi-classical Vlasov- 
LDA are reliable approximations to the fully fledged three dimensional TDLDA.

\section{Acknowledgements}

The authors thank the French-German exchange program PROCOPE number 99074 and Institut Universitaire de France for financial support during the realization of this work.

\section{References and Notes}

1. de Heer, W. A. Rev. Mod. Phys. 1993, 65, 611

2. Brack, M. Rev. Mod. Phys. 1993, 65,677

3. Clusters of atoms and molecules, 1994, Ed. by Haberland, H., Springer Ser. in Chem. Phys., vol. 52 , Springer, Berlin

4. Metal clusters, 1999, Ed. by Ekardt, W., Wiley, New-York

5. Chandezon, F.; Guet, C.; Huber, B. A.; Jalabert, D.; Maurel, M.; Monnand, E.; Ristori, C.; Rocco, J. C. Phys. Rev. Lett. 1995, 74, 3784

6. Schlipper, R.; Kusche, R.; von Issendorff, B.; Haberland, H. Phys. Rev. Lett. 1998, 80, 1194

7. Näher, U.; Bjornholm, S.; Frauendorf, S.; Garcias, F.; Guet, C. Phys. Rep. 1997, 285, 245

8. Bréchignac, C.; Cahuzac, Ph.; Carlier, F.; de Frutos, M. Phys. Rev. Lett. 1990, 64, 2893

9. Density Functional Theory: An Approach to the Quantum Many-Body Problem, 1990, Dreizler, R. M.; Gross, E. K.U., Springer, Berlin

10. Calvayrac, F.; Reinhard, P.-G.; Suraud, E. Ann.Phys.(N.Y.), 1997, 255, 125

11. Ullrich, C.; Reinhard, P.-G.; Suraud, E. J. Phys. B 1997, 30, 5043

12. Reinhard, P.-G.; Suraud, E. Euro. Phys. J. D 1998, 3, 175

13. Ullrich, C.; Reinhard, P.-G.; Suraud, E. Euro. Phys. J. D 1998, 1, 303

14. Gross, M.; Guet, C. Z. Phys D 1995, 33, 289

15. Yabana, Y.; Bertsch, G. F. Phys. Rev. B 1996, 26, 4199

16. Calvayrac, F.; Reinhard, P.-G.; Suraud, E. J. Phys. B 1998, 31, 5023

18. Plagne, L.; Guet, C. Phys. Rev. A 1999, 59, 4461

19. Ullrich, C.; Reinhard, P.-G.; Suraud, E. J. Phys. B 1998, 31, 1871

20. Yabana, K.; Tazawa, T.; Abe, Y.; Bozek, P. Phys. Rev. A 1998, 57, R3165

21. Perdew, J. P.; Wang, Y. Phys. Rev. B 1992, 45, 13244

22. Féret, L.; Suraud, E.; Calvayrac, F.; Reinhard, P.-G. J. Phys. B 1996, 29, 4477

23. Reinhard, P.-G.; Genzken, O.; Brack, M. Ann.Phys. (Leipzig) 1996, 5, 576

24. Brack, M.; Guet, C.; Hakansson, H.-B. Phys.Rep. 1985, 123, 275

25. Calvayrac, F.; Domps, A.; Reinhard, P.-G.; Suraud, E.; Ullrich, C. Euro. Phys. D 1998, 4, 207

(C) 2000 by MDPI (http://www.mdpi.org). 